\title{
Executive function in depression: the role of performance strategies in aiding depressed and non-depressed participants
}

\author{
S Channon, P S S Green
}

\begin{abstract}
Objectives-Depression has been found to be associated with dysfunction in executive processes, whereas relatively automatic processes are thought to remain intact. Failure to generate or implement adequate performance strategies has been postulated in depressed participants. The present study investigated spontaneous strategy usage in depressed and control participants, and the effectiveness of providing a hint about performance strategies.

Methods-Unipolar depressed participants were compared with matched healthy controls on three tasks sensitive to executive function: memory for categorised words, response suppression, and multiple scheduling. Participants in each group were randomly allocated to strategy aid and no strategy aid conditions. Those in the strategy aid condition were given a hint about the use of an appropriate performance strategy for each task, in addition to the standard instructions given to those in the no strategy aid condition.
\end{abstract}

Results-Depressed participants performed worse than controls on each of the three tasks, and were found to use appropriate performance strategies less often. Provision of strategy hints increased the use of performance strategies in two of the three tasks, memory for categorised words, and response suppression, but did not significantly improve overall performance for either group.

Conclusions-The findings were consistent with the view that depressed participants fail to use appropriate performance strategies spontaneously to the same extent as controls. However, provision of information alone does not seem to be an adequate means of enhancing performance. The role of performance strategies in cognitive impairment in depression is discussed, both in terms of initiating use of such strategies and carrying these out efficiently.

(F Neurol Neurosurg Psychiatry 1999;66:162-171)

Correspondence to:

Dr S Channon,

Subdepartment of Clinical

Health Psychology, UCL

Gower Street, London

WC1E 6BT, UK, email

S.Channon@ucl.ac.uk

Received 20 January 1998 and in revised form

21 August 1998

Accepted 11 September 1998 ter changes. Evidence of altered regional cerebral blood flow (rCBF) in both resting and activation studies has been reported in depressed patients in the cingulate, prefrontal cortex, and subcortical structures, ${ }^{12}$ and induction of dysphoric mood has also been found to produce $\mathrm{rCBF}$ changes in the prefrontal cortex. $^{3}$ Cognitive impairment in depressed participants has been linked to specific rCBF abnormalities, especially in the medial prefrontal cortex. ${ }^{4}$ The frontal lobes have been linked to the supervisory attentional system. ${ }^{5}$ This is thought to be functionally equivalent to the central executive component of working memory, a limited resource information processing system. ${ }^{6}$ The central executive is thought to be responsible for the allocation of attentional resources in the processing and manipulation of information in executive operations, coming into play to deal with novelty in problem solving situations. Depression has been associated with impairment in the central executive component of working memory. ${ }^{7-9}$ Alterations in neurotransmitter activities and in levels of arousal have been put forward to explain impaired processing in depression at a physiological level. ${ }^{10}$ At a cognitive level, attention has focused on the role of dysfunctional negative thoughts in partially occupying central executive resources and thereby mediating cognitive impairments, ${ }^{11}$ although Watts ${ }^{12}$ pointed out that these may not always be the key factor accounting for poor performance on all tasks.

Impairments in everyday cognitive function are commonly reported by those with depression, sometimes to the point when dementia rather than depression is suspected. There is growing evidence of depressive impairments on tests sensitive to executive dysfunction. Studies that have used traditional neuropsychological tests have in some instances shown impairment in clinically depressed or dysphoric participants on measures such as the trail making test, ${ }^{13}$ serial addition (PASAT), ${ }^{7}$ the Stroop colour-word test, ${ }^{14}$ letter fluency, ${ }^{14}{ }^{15}$ semantic fluency, ${ }^{16}$ the Tower of London planning test, ${ }^{217}$ and the Wisconsin card sorting test. ${ }^{9}$ Evidence of depressive or dysphoric impairment has also been reported on other executive tests such as subtests from the CANTAB battery, ${ }^{18}$ syllogisms, ${ }^{8}$ fault diagnosis, ${ }^{19}$ and concept formation. ${ }^{20-22}$ Executive processes are also likely to contribute to impairments in memory performance, as depressed participants have been found to show greater impairment in recalling information that benefits 
from semantic organisation compared to information that does not benefit greatly, ${ }^{23}$ although executive deficits involving frontosubcortical dysfunction may not account fully for memory impairments in depression. ${ }^{18}$ An understanding of both the nature of cognitive impairments in depression and of the effectiveness of remedial methods has important clinical implications, especially since the evidence suggests that cognitive impairments associated with depression do not always remit after clinical recovery. ${ }^{24}$

Processing resource accounts of depression ${ }^{10} 1125-28$ predict that performance deficits should occur on tasks involving controlled processes if task demands exceed available resources, whereas tasks that are processed automatically are thought to make only minimal demands on these limited attentional resources. Models differ in the extent to which reduced cognitive resources are seen to be invariant, or flexible and responsive to environmental cues. The evidence does not seem to support the notion of a simple linear relation between increasing task difficulty and depressive impairment, as depressed participants do not reliably show impairment across tasks as level of difficulty increases. The cognitive initiative account of depression ${ }^{28}$ suggests that impairments will be maximal when tasks potentially benefit from performance strategies, but participants are not required to make use of them. Both cognitive and motivational factors might potentially contribute to depressive impairments in strategy usage. In relation to motivational accounts, attempts to ameliorate depressive impairments by manipulating incentives ${ }^{29}$ have not proved fruitful, and Baker and Channon ${ }^{22}$ found no differences in response to predetermined negative and positive feedback in dysphoric participants. However, there is some supporting evidence. For instance, Elliott et $a l^{18}$ found that depressed participants were more likely than other patient groups to fail the next item given feedback about an error on the previous item, which the authors interpreted as either a negative response to failure, or failure to monitor and adjust their performance adequately in response to feedback.

When approaching a novel situation, performance often benefits from approaching it in a systematic manner, monitoring the effect of each manoeuvre, and evaluating success or failure in planning the next steps, requiring complex manipulation and updating of the available information. Strategies for doing this may draw on previous experience, although the links between the novel situation and prior experience may be tenuous, requiring active search through stored memories and knowledge. Depression may reduce people's ability to generate such performance strategies, or to carry these out efficiently, and presumably the nature and extent of any impairment will depend on the particular task characteristics. Hertel and Hardin ${ }^{28}$ found that induced or naturally occurring dysphoric mood was associated with poorer recognition of words presented in an earlier spelling test, but that provision of a strategy at recognition aided performance, and that this effect tended to be greater for the naturally occurring dysphoric group. Hertel and Rude ${ }^{30}$ showed that the use of a very simple strategy, repeating the target word at the end of the trial to focus attention on the task in hand, was sufficient to ameliorate depressive impairments in memory performance. In another study ${ }^{31}$ provision of a contextual cue (word pairs) facilitated the recollection aspect of recognition memory performance for both depressed and control participants. On reasoning tasks, the findings seem less straightforward. Baker and Channon ${ }^{22}$ found that focusing participants' attention on the task at the critical stages was not sufficient to ameliorate dysphoric impairment on relatively complex concept formation problems. However, Hertel and Knoedler ${ }^{32}$ reported that giving a hint in relation to solving logic problems impaired the performance of control participants, but not that of dysphoric patients.

In general, the extent to which the provision of performance strategies is effective in ameliorating any depressive or dysphoric impairment to normal levels is likely to be influenced by the complexity of the task. It seems reasonable to suppose that a memory recall task may benefit from increased rehearsal at the encoding stage, such as the Hertel and Rude ${ }^{30}$ manipulation of repeating the target word, if such rehearsal is absent or falls below a certain minimal level. Even with a simple strategy of this nature, there are ways in which such rehearsal may function to improve performance. For instance, it may serve simply to increase the allocation of attentional resources to task performance, or might lead more specifically to the use of a semantic encoding strategy. In the case of more complex problem solving tasks, which often make multiple concurrent demands on attentional resources, manipulations that simply increase the focus of attention on task performance may be insufficient to facilitate usage of complex strategies necessary for effective performance. ${ }^{22}$ To facilitate performance, it is presumably necessary but not always sufficient to focus sufficient attentional resources on the problem.

Further study of executive functioning in depression has implications both for diagnosis and management of the disorder. The present study was designed to examine the spontaneous use of performance strategies in executive tasks in clinically depressed participants, and additionally to assess the value of providing information about performance strategies in aiding depressive performance. Tasks were selected that were known to be sensitive to executive impairment in participants with frontal lobe lesions, and that potentially benefited from an appropriate performance strategy. These were memory for categorised words, ${ }^{33}$ and known to be sensitive to depression $^{23}$; response suppression (based on the Hayling test ${ }^{34}$; and multiple task scheduling (based on the six elements test ${ }^{35}$ ). Depressed and control participants were randomly assigned to one of two conditions. Those in the no strategy aid condition performed the tasks with standard instructions, whereas those in 
the strategy aid condition were given a hint for each task of an appropriate performance strategy. It was predicted that depressed participants would be poorer than controls on each task in the no strategy aid condition, and that this would be linked to less efficient use of appropriate performance strategies in the depressed participants. Those in the strategy aid condition were expected to show better performance than those in the no strategy aid condition, and the study aimed to explore whether depressed participants were differentially aided compared with controls by the strategy hints.

\section{Methods}

DESIGN

Two groups of participants, clinically depressed and controls, were each randomly allocated to one of two conditions that differed only in terms of whether a strategy prompt was given after the task instructions (strategy aid or no strategy aid). All participants carried out the same three experimental measures in one of the two conditions.

\section{EXPERIMENTAL MEASURES}

In the no strategy aid condition, participants performed the tasks under standard instructions. In the strategy aid condition, they were given an additional instruction for each task designed to facilitate the use of an appropriate performance strategy. For each task, there were two equivalent sets of materials, and material set was counterbalanced within each group for each condition.

Memory for categorised words task

This task was based on that of Channon et al. ${ }^{33}$ For the initial study phase, 16 words were presented one at a time on a computer screen at a rate of one every 2 seconds. The words belonged to four superordinate categories, each containing four words; all the words were presented in a fixed randomised order. The categorised words were concrete, neutral nouns matched for length, number of syllables, and word frequency. ${ }^{36}$ All participants were instructed to try and remember these words, as they would be asked to write them down afterwards. Participants in the strategy aid condition were additionally told: "The words in the following list can be put into several groups of related words. Use this to help you to remember them". This prompt was on display throughout the task.

After initial list presentation, participants were asked to write down as many words as they could remember, in unlimited time. They then completed an interference task for $90 \mathrm{sec}-$ onds, and were given a 32 item recognition test, presented one at a time on the screen. Sixteen of these 32 items were words from the previous study phase, and 16 were matched distractors; the words were presented in a fixed randomised order. Half of the distractors came from the same categories as the test items, and half were drawn from two new categories. At the end of the session, after completing the other experi- mental tasks, participants were again asked to write down all the words they could remember from the initial study list.

\section{Response suppression task}

This was based on the Hayling test, ${ }^{34}$ and consisted of two parts, each containing 15 sentences referring to everyday events. The last word of each sentence was omitted. The sentences were presented verbally, one at a time, by the experimenter, and two initial practice sentences were used for each part. For both parts, the instructions specified that the sentences should be completed as quickly as possible. For the first part of the task, participants in both the strategy aid and no strategy aid conditions were instructed to complete the sentence with a word that could fit as quickly as possible. For the second part of the task, participants were instructed to complete the sentence with a word that made "no sense at all in the context of the sentence". They were told that the word should be "unrelated to the sentence in every way". The task thus involved inhibiting straightforward sentence completions, to find a nonsensical ending word. Participants in the strategy aid condition were given a prompt which remained on display throughout the task: they were told to imagine that they were on a holiday and to think of words relating to this to complete the sentence. All participants were corrected on the practice sentences if they mistakenly completed a sentence with a related word.

Burgess and Shallice ${ }^{34}$ found that participants tended to complete sentences in the response suppression task with words that were either office related, from looking around the room, or generated from other semantic categories. In the present study, responses were scored according to the number of nonsensical completions derived from semantic categories, whether office related or from other semantic categories - for example, she called the husband at his "pencil". For the strategy aid participants, holiday related words were treated similarly as derived from a semantic category. Errors were scored for words that were either straightforward sentence completions - for example, she called the husband at his "office", or were semantically related to the sentences-for example,"phone" or "wife".

\section{Multiple scheduling task}

This task was based on the six elements test ${ }^{35}$; the number of tasks used was increased from six to eight, and the time limit reduced, to increase the difficulty level. It consisted of a set of eight open ended pen and paper tasks that participants were instructed to complete, subdivided into two sets of four: mirror reading, identifying the position of words, object knowledge questions, and drawing objects. In the mirror reading tasks, participants were presented with a series of 175 back to front words and asked to write the words the correct way round. In the word position task, participants were given a page consisting of words presented in columns, and a series of 176 positions (for example, 2nd, 134th, 12th), 
and were asked to identify and write down the words on the page that occurred in these positions. For the object knowledge questions, participants were presented with a series of 138 written questions asking where various objects would be commonly found (for example, where would you find a keyhole?). For the drawing task, participants were given blank pages and were asked to draw the basic outline of various common objects (for example, items of clothing).

Each task was explained in turn. Participants were told that there were four different tasks, and that each task had two sections, A and B, making eight tasks for them to complete in total. These were laid out in a four by two matrix on a large table. They were told that they would be given 10 minutes in all. They were correctly informed that they would not be able to complete everything in 10 minutes, and that it was more important to attempt at least part of each task than to complete any single task. In addition, they were given two rules. Firstly, they were told that they should not do a section A and a section B of the same task, one after the other. Secondly, they were told that they must not do a section A of one task, followed by a section A of another task. The instructions were on display to participants throughout the task. Those in the strategy aid condition were given a strategy prompt (also on display throughout the task): they were told to "work through the tasks alternating the A and B sections", and to "work through the tasks from left to right (tasks 1-4), although the second time you can't start with task 1 ". After the test had been explained fully, participants were then asked to outline all the instructions, and were corrected if their understanding was insufficient. Once they had started the tasks, they were informed when they had 5 minutes, 2 minutes, and 30 seconds left of the 10 minutes, and could also check the remaining time at any other point.

PARTICIPANTS AND PROCEDURE

DSM-III-R ${ }^{37}$ was used to select participants who met diagnostic criteria for major depressive disorder, based on ratings from interviews using the SCID (structured clinical interview for DSM-IIIR ${ }^{38}$ ). The Beck depression inventory ${ }^{39}$ and the Hamilton rating scale $^{40}$ were also administered. Those with a history of bipolar disorder were excluded. General exclusion criteria were a vocabulary score more than one SD below average on the spot the word test, ${ }^{41}$ any relevant history of physical or neurological illness, alcohol or drug dependence, mania, hypomania, or other psychiatric disorder, or electroconvulsive treatment within the past 12 months. They were recruited from a depression self help group, and from volunteer notices. The depressed group consisted of 23 participants, and 13 of these were taking antidepressant medication at the time of testing; it was not considered ethical to withdraw them from their medication outside a hospital environment. Similar exclusion criteria were used to select 23 non-depressed control participants who were recruited from volunteer notices. The controls were matched to the depressed participants in age, years of education, and vocabulary knowledge (spot the word test).

Twelve depressed (five male, seven female) and 12 control (five male, seven female) participants were randomly allocated to the strategy aid condition. $t$ Tests showed that the groups did not differ significantly $(p>0.1)$ for age (depressed 41.3 (SD 13.1) years; control 41.2 (SD 11.2) years), years of education (depressed 12.8 (SD 2.6) years; control 13.4 (SD 2.4) years, or spot the word performance (depressed 12.3 (SD 2.6); control 13.0, (SD 1.9). Six of the depressed participants were on antidepressant medication (two on tricyclic drugs, two on selective serotonin reuptake inhibitor (SSRI), one on SSRI+carbamazepine, and one on a monoamine oxidase inhibitor MAOI)). The depressed group scored significantly higher $(p<0.0001)$ on the Beck depression inventory (depressed 21.4 (SD 7.1); control 3.8 (SD 2.4)). Eleven depressed (six male, five female) and 11 control (seven male, four female) participants were randomly allocated to the no strategy aid condition. $t$ Tests showed that the groups did not differ significantly $(p>0.1)$ in terms of age (depressed 35.1 (SD 11.9) years; control 38.3 (SD 14.3) years), years of education (depressed 14.4 (SD 2.3) years; control 14.3 (SD 1.9) years), or spot the word performance (depressed (SD 2.5) 13.6; control 14.5 (SD 2.4)). Seven of the depressed participants were on antidepressant medication (five on SSRIs, one on SSRI+tricyclic, one on tricyclic $+\mathrm{MAOI})$. The depressed group scored significantly higher $(\mathrm{p}<0.0001)$ on the Beck depression inventory (depressed 24.3 (SD 6.9), control 4.1 (SD 2.2)). A comparison between the two depressed groups showed that they did not differ significantly in any of the characteristics described above, nor on the Hamilton rating scale (strategy aid depressives 18.1 (SD 5.8), no strategy aid depressives 20.3 (SD 4.6)); nor did the two control groups differ significantly on any of the measures.

Participants were initally given an information sheet to read about the study, and were given an opportunity to ask questions. They were assured that all details given would remain confidential. They then were asked to sign a consent form to say that they agreed to take part. All participants then received the SCID interview, and a detailed history was taken to screen out physical or neurological illness or injury. They filled out the depression questionnaire, and then carried out the experimental tasks as described above. The word list task was always administered at the start of the experimental session, to allow time for delayed recall; the other two tasks were presented in counterbalanced order within each group for each condition.

\section{Results}

EXPERIMENTAL TASKS

Mean scores (SD) for the groups in the strategy aid and no strategy aid conditions are shown in the table. A significance level of $5 \%$ was adopted throughout. 
Memory for categorised words task

Recall of the words was examined using repeated measures analysis of variance (ANOVA) with two between group factors (group: depressed $v$ control; condition: strategy aid $v$ no strategy aid) and one within group factor (time of recall: immediate $v$ delayed). This showed a significant effect of group $(F=8.40, \mathrm{df}=1,41, \mathrm{p}=0.006)$, as depressed participants in both conditions (strategy aid and no strategy aid) performed worse than controls in immediate and delayed recall of the categorised words. There were no significant effects for strategy aid $(F=0.02, \mathrm{df}=1,41, \mathrm{p}=0.884)$, time of recall $(F=0.24, \mathrm{df}=1,41, \mathrm{p}=0.625)$, strategy $\times$ time $(F=0.02, \mathrm{df}=1,41, \mathrm{p}=0.884)$, group $\times$ strategy $(F=0.24, \mathrm{df}=1,41, \mathrm{p}=0.625)$, or group $\times$ strategy $\times$ time $(\mathrm{F}=2.03, \mathrm{df}=1,41$, $\mathrm{p}=0.162$ ). A similar ANOVA examining the number of categories used in free recall (maximum possible four) showed a significant group $\times$ time effect $(F=5.93, \quad \mathrm{df}=1,41$, $\mathrm{p}=0.019)$, and no other significant effects, using logarithmically transformed scores. Depressed participants recalled words from fewer categories than controls at delayed recall $(t=2.15, \mathrm{df}=24.3, \mathrm{p}=0.042)$, but did not differ at immediate recall $(t=0.41, \mathrm{df}=43, \mathrm{p}=0.682)$, when most participants from both groups recalled words from all four categories. Recognition memory for the words at the end of the test also showed a significant main effect of group $(F=7.56, \mathrm{df}=1,41, \mathrm{p}=0.009)$, but no significant effect of strategy $(F=1.00, \mathrm{df}=1,41$, $\mathrm{p}=0.323)$ or group $\times$ strategy interaction $(F=0.47, \mathrm{df}=1,41, \mathrm{p}=0.499)$. The control participants were close to ceiling in performance for recognition of the categorised words.

To investigate the use of semantic categorisation in retrieval, the order in which participants listed words was also examined. Immediate and delayed free recall lists were divided into "ordered" words which were clustered in categories (two or more words in category order) and "'non-ordered" words (those which appeared singly and were not followed by another word from the same category). Repeated measures ANOVA comparing performance on the ordered words alone for immediate and delayed recall showed that the depressed participants produced significantly fewer "ordered" words than controls $(F=6.71, \mathrm{df}=1,41$, $\mathrm{p}=0.013$ ), using logarithmically transformed scores. The effect of strategy aid was also significant $(F=6.75, \mathrm{df}=1,41, \mathrm{p}=0.013)$, showing that those in the strategy aid condition recalled more ordered words than those in the no strategy aid condition; there were no significant interactions involving group or strategy aid. Participants were also compared on the number of "non-ordered" words, using logarithmically transformed scores. This showed no significant difference between the depressed and control groups $(F=0.68, \mathrm{df}=1,41$, $\mathrm{p}=0.414)$, and no significant effects involving group; there was a significant effect of strategy aid $(F=8.02, \mathrm{df}=1,41, \mathrm{p}=0.007)$, showing that participants in the no strategy aid condition recalled more non-ordered words than those in the strategy aid condition. Taken together, the findings suggest that strategy aid instructions increased the use of semantic categories to order word recall, but did not necessarily increase the overall number of words recalled.

\section{Response suppression task}

An ANOVA with two between group factors (depressed $v$ control; strategy aid $v$ no strategy aid) was used to examine the number of inhibitory errors ( straightforward or semantically related completions) made in nonsensical sentence completion was carried out, using a logarithmic transformation. The depressed participants made significantly more errors than controls $(F=5.17, \mathrm{df}=1,41, \mathrm{p}=0.028)$, but the effect of strategy aid was not significant

Performance means SD for the categorised word task, response suppression task, and multiple scheduling task

\begin{tabular}{|c|c|c|c|c|}
\hline & \multicolumn{2}{|l|}{ Depressed } & \multicolumn{2}{|l|}{ Controls } \\
\hline & $\begin{array}{l}\text { Strategy aid } \\
\left(n=12^{\star}\right)\end{array}$ & $\begin{array}{l}\text { No strategy aid } \\
(n=11)\end{array}$ & Strategy aid $(n=12)$ & $\begin{array}{l}\text { No strategy aid } \\
(n=11)\end{array}$ \\
\hline \multicolumn{5}{|l|}{ Categorised word task } \\
\hline \multicolumn{5}{|l|}{ Correct responses: } \\
\hline Immediate recall/16 & $9.36(1.91)$ & $9.45(2.81)$ & $11.67(1.56)$ & $11.45(1.69)$ \\
\hline Delayed recall/16 & $9.00(2.49)$ & $9.82(3.71)$ & $11.83(2.66)$ & $10.73(2.65)$ \\
\hline Recognition/32 & $28.45(2.58)$ & $28.27(2.37)$ & $30.42(1.16)$ & $29.45(1.13)$ \\
\hline \multicolumn{5}{|l|}{ Categories recalled/4: } \\
\hline Immediate recall & $3.91(0.30)$ & $3.91(0.30)$ & $3.83(0.39)$ & $3.91(0.30)$ \\
\hline Delayed recall & $3.55(0.69)$ & $3.73(0.65)$ & $3.92(0.29)$ & $4.00(0.00)$ \\
\hline \multicolumn{5}{|l|}{ Ordered words: } \\
\hline Immediate recall & $7.74(2.73)$ & $5.18(4.67)$ & $10.25(3.31)$ & $7.45(3.88)$ \\
\hline Delayed recall & $7.55(3.08)$ & $7.00(4.88)$ & $10.75(3.67)$ & $8.45(3.67)$ \\
\hline \multicolumn{5}{|l|}{ Non-ordered words: } \\
\hline Immediate recall & $1.73(2.53)$ & $4.27(3.77)$ & $1.50(2.50)$ & $4.00(3.44)$ \\
\hline Delayed recall & $1.45(1.69)$ & $2.82(2.04)$ & $1.08(1.51)$ & $2.36(2.46)$ \\
\hline \multicolumn{5}{|l|}{ Response suppression task } \\
\hline \multicolumn{5}{|l|}{ Nonsensical sentences: } \\
\hline No of errors & $3.45(2.98)$ & $3.45(2.38)$ & $2.17(2.76)$ & $1.64(1.50)$ \\
\hline Categorised completions & $9.45(5.57)$ & $6.09(4.39)$ & $12.75(2.99)$ & $8.82(4.33)$ \\
\hline Total time & $54.36(32.47)$ & $43.00(31.85)$ & $34.67(19.37)$ & $20.82(8.06)$ \\
\hline \multicolumn{5}{|l|}{ Straightforward sentences: } \\
\hline Total time & $19.82(4.14)$ & $25.18(17.71)$ & $18.58(3.65)$ & $19.09(2.39)$ \\
\hline \multicolumn{5}{|l|}{ Multiple scheduling task } \\
\hline Different tasks attempted/8 & $6.58(2.11)$ & $6.27(1.95)$ & $7.33(1.56)$ & $7.64(1.21)$ \\
\hline No of rule breaks & $1.25(1.29)$ & $0.45(0.69)$ & $0.67(0.98)$ & $0.55(0.93)$ \\
\hline Efficiency score/8 & $4.08(2.54)$ & $3.82(2.36)$ & $5.50(2.58)$ & $4.27(3.32)$ \\
\hline
\end{tabular}

*As a result of an adminstrative error, one participant in this group did not do the memory task, and another did not do the response suppression task. 
( $F=0.00, \mathrm{df}=1,41, \mathrm{p}=0.943$; nor was the group by strategy aid interaction significant $(F=0.12$, $\mathrm{df}=1,41, \mathrm{p}=0.729$ ).

To investigate strategy usage by the two groups further, solutions were scored for the number of completions belonging to a semantically related category (whether holiday items or other categories, such as items belonging to an office). A similar ANOVA showed a significant effect of group, such that the depressed participants gave significantly fewer categorised completion words $(F=5.29, \mathrm{df}=1,41$, $\mathrm{p}=0.027$, and a significant effect of condition, such that participants in the strategy aid condition gave more categorised completions $(F=7.76, \mathrm{df}=1,41, \mathrm{p}=0.008)$; the interaction between group and strategy aid was not significant $(F=0.05, \mathrm{df}=1,41, \mathrm{p}=0.829)$. Time taken to find nonsensical sentence completions was also examined, using logarithmically transformed scores. There was a significant effect of group $(F=8.71, \mathrm{df}=1,41, \mathrm{p}=0.005)$, as the depressed participants were markedly slower than controls. The effect of strategy aid was also significant $(F=4.92, \mathrm{df}=1,41, \mathrm{p}=0.032)$; the group by strategy aid interaction was not significant $(F=0.18, \mathrm{df}=1,41, \mathrm{p}=0.674)$. Thus, provision of the holiday word strategy increased the use of categorised words for both the depressed and control groups, but also slowed performance. The depressed participants in both the strategy aid and no strategy aid conditions achieved fewer correct completions than controls, took longer to do so, and were less likely to use a semantic strategy.

A $t$ test showed that speed of performance did not differ significantly between the depressed and control groups when they were compared on the straightforward sentence completions ( $t=1.33, \mathrm{df}=23.37, \mathrm{p}=0.204)$; no strategy manipulation was used for this initial part of the task.

Multiple scheduling task

ANOVA with two between group factors (depressed $v$ control; strategy aid $v$ no strategy aid) was used to compare how many of the eight different tasks the two groups attempted. There was a significant main effect of group $(F=4.20, \mathrm{df}=1,42, \mathrm{p}=0.047)$; the effect of strategy was not significant $(F=0.00, \mathrm{df}=1,42$, $\mathrm{p}=0.994)$, nor the group $\times$ strategy interaction $(F=0.35, \mathrm{df}=1,42, \mathrm{p}=0.555)$. Depressed participants in both groups performed worse than controls, whose performance was very close to ceiling. All but three of the control participants $(87 \%)$ attempted all eight of the tasks, whereas only 12 of 23 depressed participants (52\%) attempted all eight tasks. All but one of the participants who did not attempt all eight tasks also attempted fewer than eight tasks overall, and eight of the 11 depressed participants in this category did not visit any tasks more than once, suggesting that their difficulties lay primarily in shifting set as often as required. A similar ANOVA was used to compare the groups on the number of rule breaks involving order-that is, moving from the A version of a task directly to the $\mathrm{B}$ version, or vice versa, using logarithmically transformed scores. Er- rors of order were relatively uncommon, and the groups did not differ significantly on these $(F=0.77, \mathrm{df}=1,42, \mathrm{p}=0.386)$; the effect of strategy was not significant $(F=2.25, \mathrm{df}=1,42$, $\mathrm{p}=0.141)$, nor the group $\times$ strategy interaction $(F=1.11, \mathrm{df}=1,42, \mathrm{p}=0.298)$.

To examine further the efficiency of the strategies used by the groups, an efficiency score was derived by examining the order in which the tasks were performed, and counting the number of moves $(0-8)$ which followed the most salient and efficient strategy (which was also the one cued in the strategy aid condition), without any deviations. This strategy involved starting on one side (for example, left), and moving from one side to the other (for example, left to right), alternating $\mathrm{A}$ and $\mathrm{B}$ tasks, on the first four moves (for example, 1A, $2 \mathrm{~B}, 3 \mathrm{~A}, 4 \mathrm{~B})$; and then following one of two types of pattern for the second four moves (for example, $2 \mathrm{~A}, 3 \mathrm{~B}, 4 \mathrm{~A}, 1 \mathrm{~B}$ or $3 \mathrm{~B}, 4 \mathrm{~A}, 1 \mathrm{~B}, 2 \mathrm{~A})$. An ANOVA with two between group factors (depressed $v$ control; strategy aid $v$ no strategy aid) showed that differences between the groups in the efficiency of their strategy on this measure were not significant $(F=1.36$, $\mathrm{df}=1,42, \mathrm{p}=0.250$ ), and the strategy aid hint did not have a significant effect on performance $(F=0.87, \mathrm{df}=1,42, \mathrm{p}=0.357)$; nor was there a significant group $\times$ strategy aid interaction $(F=0.36, \mathrm{df}=1,42, \mathrm{p}=0.552)$. Only three of the depressed participants $(13 \%)$ and eight of the control participants $(35 \%)$ scored maximum on this measure. One additional depressed participant also visited all eight tasks without repetition or order errors, but started in the middle of the tasks rather than from left or right. All the remaining participants who visited all eight tasks without order errors repeated one or more of these tasks before sampling all eight, a strategy not forbidden by the rules, but arguably less efficient.

INTERACTIONS BETWEEN THE TASKS

To examine participants' performance across tasks, intercorrelations were calculated between the measures that significantly differentiated the groups for each of the three tasks, as follows: memory for categorised words (number of words recalled; number of categories used at delayed recall; number of words recognised; number of ordered words); response suppression (number of categorised completion words; number of errors; time taken to make nonsensical sentence completions); multiple subgoal scheduling (how many of the eight tasks were attempted). For the depressed group, Spearman rank correlation coefficients showed no significant correlations between the memory task and the other two tasks, although there were significant intercorrelations between different memory measures (recall and number of categories $r=0.53$, $\mathrm{p}=0.011$; recall and recognition $\mathrm{r}=0.82$, $\mathrm{p}=0.0001$; recall and number of ordered words $r=0.75, \mathrm{p}=0.0001$; categories and recognition $r=0.59, \mathrm{p}=0.004$; recognition and ordered words $r=0.67, \mathrm{p}=0.001)$. There was a significant negative correlation between the number of tasks attempted on the multiple subgoal 
scheduling task, and the number of errors on the response suppression task $(r=-0.67$, $\mathrm{p}=0.001)$. There was also an intercorrelation between the number of response suppression errors and time taken on the response suppression task $(r=0.45, \mathrm{p}=0.034)$. For the control group, the pattern was similar. Memory performance did not correlate significantly with the response suppression task, but there were significant intercorrelations between different memory measures (recall and recognition $r=0.52, \mathrm{p}=0.011$; recall and number of ordered words $r=0.73, \mathrm{p}=0.0001$; recognition and ordered words $r=0.56, p=0.006)$. Correlations beteen the number of tasks attempted on the multiple subgoal scheduling task and the other two tasks were not carried out, as nearly all participants in the control group were at ceiling on the multiple scheduling task.

CORRELATIONS WITH MOOD VARIABLES

For the depressed participants, Spearman rank correlations were also examined between the measures that significantly differentiated the groups and variables relating to severity of depressive illness, namely Beck depression inventory score, Hamilton score, and presence of antidepressant medication. None of the correlations between task performance and severity of illness reached significance for the depressed participants. $t$ Tests comparing medicated and non-medicated depressed participants on the same performance measures also showed no significant differences.

\section{Discussion}

The depressed group showed impairment relative to controls on each of the three experimental tasks. Detailed examination of qualitative aspects of performance suggested that the depressed participants also made less spontaneous use of appropriate strategies for task performance. The provision of a strategy aid in the form of information about a specific performance strategy for each task was associated with significantly increased use of the strategy for two of the three tasks for both depressed and control participants in the strategy aid versus no strategy aid condition. However, increased strategy usage did not lead to significantly improved overall performance for either the depressed or control participants.

Memory for categorised words presented in pseudorandomised order was expected to be more efficient if participants made use of semantic categorisation to encode and retrieve these words. The findings suggest that the control participants in both the strategy aid and no strategy aid conditions made use of such categorisation more than the depressed participants, as their free recall of the words contained higher numbers of words ordered by semantic category. At delayed recall, control participants were more likely to recall words from each of the four semantic categories than depressed participants, and this might also have reflected a greater tendency to use categorisation as a cue for recall. Depressed participants have previously been found to be selectively impaired in memory for categorised words when these are presented in randomised rather than semantically ordered fashion. ${ }^{23}$ The findings are also consistent with evidence from both lesion studies $^{42} 43$ and PET studies ${ }^{44}$ which suggest that areas of the frontal lobes may play a specific part in strategic aspects of memory encoding and retrieval. In the present study, control participants were also better at recognising the words correctly, suggesting that their superior performance was not solely attributable to a more efficient retrieval process in the more effortful recall condition, but probably involved more effective elaborative encoding processes. Because the recognition test was given after the immediate and delayed recall tests, this cannot be seen as a pure measure of recognition memory. Impairment in recognition memory is not always reported in depression, and is known to be a less sensitive measure than free recall. ${ }^{45}$ However, Hertel and Milan, ${ }^{31}$ using a process dissociation procedure, identified impairment in the recollection but not the familiarity component of recognition memory in depression, consistent with the hypothesis of impairment in controlled processes in depression.

Giving a suggestion that participants should put together the related words (the strategy aid hint) was associated with differences in the use of categorisation in recall for both the depressed and control groups. Those in the strategy aid condition recalled more words ordered by category, and fewer words not ordered by category, than those in the no strategy aid condition. The hint thus seemed to be effective at least to some extent in cueing the use of a semantic strategy. Presumably the hint was informative even to participants who would naturally have looked for strategies such as semantic organisation, as it made them aware of the potential for organising the words semantically at the beginning of the task, rather than discovering it at a later point. However, although both immediate and delayed recall and recognition means for the strategy aid controls were higher than for the no strategy aid controls, these differences did not reach significance, and there was no evidence of improved overall recall or recognition performance for the depressed participants. Ceiling effects may have limited the sensitivity of these measures to the strategy aid manipulation, at least for the control group. Semantic encoding strategies have been found to benefit participants with frontal lesions. For instance, participants with left frontal lobe lesions differed from those with left temporal lobe lesions in one study, ${ }^{42}$ as the frontal but not the temporal group was found to improve to normal levels when presentation of the material was organised by semantic categories, and category names were provided for encoding and retrieval. In another study, Gershberg and Shimamura $^{43}$ (experiment 3 ) reported that participants with frontal lobe lesions benefited from instructions to use a list of semantic categories which was provided as a cue, whether this was presented at encoding or at retrieval. By contrast with depressive participants in the present study, those using participants with 
frontal lobe lesions showed differential improvement relative to controls. This may reflect greater initial impairment in participants with frontal lesions than in the depressed participants studied here, and the strategy manipulations used also gave more help than those in the present study by providing category names in addition to instructions to use a strategy of semantic categorisation.

Depressed participants in the present study made more errors than controls in completing sentences with nonsensical words on the response suppression task, and were also slower in this part of the task, although unlike participants with structural lesions involving the frontal lobes reported by Burgess and Shallice, ${ }^{34}$ depression did not impair performance in completing sentences with sensible words, the initial part of the task that did not involve response suppression. Inspection of the number of categorised words used to complete the sentences showed that they gave fewer categorised completions than controls, suggesting that they were less likely to use a semantic strategy to generate nonsensical word completions, and may have taken longer to generate completion words when less reliant on such a strategy. The provision of a strategy aid hint that participants could complete the sentences with words related to holidays was associated with greater use of categorised words in both the depressed and control groups, but also with slower performance. Although the mean error rate was slightly lower for the strategy aid controls than for the no strategy aid controls, this difference was not significant, and there was no difference in error rates between the strategy aid and no strategy aid depressed participants. Differences between the depressed and control groups may involve both initiating and implementing efficient performance strategies, and simply providing information on an appropriate strategy is not sufficient to guarantee normal efficiency of usage. The holiday strategy may also have been slower to access than a strategy used spontaneously by many controls, that of choosing office related items by looking around the room. Moreover, in this task, a prompt to generate words from a semantic category would be unlikely to overcome all difficulties in inhibiting straightforward completion words, or production difficulties in generating words from a semantic category such as holiday items.

On the multiple scheduling task, depressed participants were again impaired compared with controls. Many of the depressed participants failed to attempt all eight of the tasks, whereas this was uncommon among the controls. Most of the participants who failed to attempt all the tasks also did not attempt any task more than once, suggesting difficulties in shifting appropriately between tasks. This may have reflected impairment in monitoring the passage of time to fit in all the tasks within the 10 minute time limit, or perseverative difficulties in generating an internal cue and shifting from one task to the other without external cues to do so. Alternatively, it may reflect differences in judgement as they may have placed less importance on the rules of the test than controls, preferring to attempt a greater proportion of the items on individual tasks. All participants were able to repeat the rules of the test before they began, and had these available visually throughout, making an explanation in terms of memory or comprehension failure in relation to the rules unconvincing. Shallice and Burgess $^{35}$ reported that two of their three participants with frontal lobe lesions attempted fewer subtests than any of their controls on their six elements test, and the third also failed to attempt all the subtests, despite numerous shifts between subtests; the frontal participants also showed qualitatively bizarre behaviour, breaking the test rules in ways which were not seen in the present depressed participants. Shallice and Burgess discussed their findings in terms of possible impairments in plan formulation or modification, the creation and triggering of markers or messages to review actions when certain future events occur, and the on line evaluation and modification of goals in the light of events.

The strategy aid hint given in this task in the present study was not found to be effective in differentially aiding performance for the strategy aid groups compared to the no strategy aid groups. The hint given was intended to guide participants in relation to the particular order of task performance they used in sampling the eight tasks. The ordering of tasks and monitoring of those which have already been visited seems to be a relevant consideration, in view of the finding that neither the control nor depressed participants tended to use the most salient and efficient order strategy for shifting between tasks. Less than half of the controls, and less than a quarter of the depressed participants, achieved a maximum efficiency score, often as a result of visiting tasks more than once before sampling all eight. However, as many depressed participants visited fewer than eight tasks in total, it seems that the principal reason for the low scores of many of the depressed participants was difficulty in shifting between tasks, and that this would therefore have been a more appropriate target for a strategy hint. For instance, it may have been more effective to suggest that participants should remember to change tasks roughly every minute to comply with the rules. Because the control participants generally had no difficulty with this, showing ceiling effects on this measure, any such strategy aid would be likely to affect the performance of depressed participants differentially, if effective.

The findings of the study are consistent with cognitive models predicting impairment in controlled processes in depression resulting from reduced or diverted attentional processing resources, although there are difficulties in determining the precise nature of such impairment. Controversy in the literature on depression has centred around the extent to which reduced cognitive resources are seen to be invariant, or flexible and responsive to environmental cues. ${ }^{10}{ }^{1125-28}$ The cognitive initiative account of depression ${ }^{28}$ suggests that impairments will be maximal on tasks that potentially 
benefit from performance strategies, when participants are not forced to use these. The present study provided support for the notion that depressive participants are impaired on tasks that potentially benefit from appropriate performance strategies, but did not attempt to identify or compare tasks of comparable difficulty that do not benefit from such strategies. Nor were participants in the present study required to use such performance strategies, although the instructions indicated that it would be helpful to do so.

Although there were significant correlations between the response suppression and multiple subgoal scheduling tasks for the depressed group, intercorrelations between performance on the memory task and the other two tasks were generally low, both for the depressed and control groups. This raises the possibility that subsets of depressed participants might show impairment in specific aspects of executive function, such as strategic aspects of memory, set shifting, and planning. However, the possible fractionation of executive functioning into separable independent subsystems is as yet an unresolved issue. As Shallice and Burgess ${ }^{46}$ point out in relation to fractionation of frontal lobe functions, inferences cannot be adequately drawn from tasks which do not use the same type of materials, as they are likely to draw on different non-frontal processes, and potentially involve different material specific frontal lobe regions in relation to working memory. They also noted that the reliability of the measures is not well established, and that many patients with frontal lobe lesions are characteristically variable in their performance over sessions. Thus, the presence of low intercorrelations between tasks reliant on different materials does not rule out the possibility that the depressed participants share a common pathway to impaired performance, such as a reduced general pool of attentional processing resources. Further work is needed comparing performance across tasks which share more common features, such as the two types of sentence completion (straightforward and nonsensical completions), only one of which involves response inhibition, required in the Hayling test.

The evidence from the present study suggests that depressed participants seemed to use similar spontaneous performance strategies to controls, but to a lesser extent. Moreover, both depressed and control participants in the no strategy aid condition seemed spontaneously to use similar semantic strategies to those supplied in the strategy aid condition, but again to a lesser extent. Both depressed and control groups in the strategy aid condition were able to draw on information about semantic categorisation in ordering recall of categorised words and in using categorised words to perform the response inhibition task. However, there was no evidence of facilitation of performance for the depressed strategy aid group, and any facilitation of performance for the control strategy aid group did not produce significant improvements compared to the no strategy aid controls. Thus compliance with the strategy aid hints was not sufficient to increase overall levels of recall significantly on the memory task, or to reduce errors significantly for the response suppression task; moreoever, it was associated with slower performance on the second task. This raises the question of whether greater use of semantic performance strategies by control participants in these two tasks was responsible for their superior performance, or simply a reflection of some other difference between the groups. It is possible that additional performance strategies were used by the control participants, less obvious to detect, which also contributed to their performance superiority, and debriefing would have been appropriate here to increase the understanding of this. However, it is more likely that provision of brief information about performance strategies is not sufficient to enhance performance adequately. It seems probable that depressive impairment cannot be attributable entirely to impairment in initiating appropriate performance strategies, but also involves impairment in using such strategies effectively.

To facilitate performance to normal levels, compensatory aids may need to go further than provision of information about strategies alone, by providing specific performance cues, practice, or possibly specific training in the use of such strategies. Providing information about specific performance strategies potentially deals with any impairments at the level of perceiving the potential usefulness of such strategies, or generating ideas for possible strategies. Information alone does not ensure that participants actually make effective use of the strategies provided, whether because they make little attempt to do so, or try and fail to achieve this successfully. Initially, attempting to make use of an unfamiliar performance strategy may increase the working memory demands of the task, and hence any gains from complying with the strategy may not be immediate, but develop over time. Repeated use of semantic categorisation in consecutive trials in memory or response suppression type tasks might thus show increasing benefit from performance strategies. In considering failure to shift set in the multiple scheduling task, it is unclear whether providing a strategy hint before beginning the task that it would be appropriate to shift set at certain times would be adequate, or whether it would be necessary to provide an external cue to shift set during task performance. However, compensatory aids that require practice and specific training for effective usage are likely to be of limited general utility, as the essence of executive impairment according to Shallice's ${ }^{5}$ model is that it arises in the face of novel, rather than familiar, situations, and increasing familiarity reduces the novelty, and potentially the difficulty, of the situation. From a practical point of view, it is much more straightforward to provide compensatory aids at the level of information alone, than to provide specific practice or training in task performance, and this in turn has implications for the everyday value of compensatory strategy aids. 
This study was supported by a grant to SC from the Leverhulme Trust. We are grateful to Kian Vakili for assistance with the data Trust. We
collection.

1 Bench CB, Friston KJ, Brown RB, et al. The anatomy of melancholia - focal abnormalities of cerebral blood flow in melancholia-focal abnormalities of cerebral bl

2 Elliott R, Baker SC, Rogers RD, et al. Prefrontal dysfunction in depressed patients performing a complex planning task: a study using positron emission tomography. Psychol Med 1997;27:931-42.

3 Baker SD, Frith CD, Dolan RJ. The interaction between mood and cognitive function studied with PET. Psychol Med 1997;27:565-78.

4 Dolan RJ, Bench CJ, Brown RG, et al. Neuropsychological dysfunction in depression: the relationship to regional cerebral blood flow. Psychol Med 1994;24:849-57.

5 Shallice T. From neuropsychology to mental structure. Cambridge: Cambridge University Press, 1988.

6 Baddeley A, Hitch GJ. Working memory. In: G Bower, ed. Recent advances in learning and motivation. Vol VII New York: Academic Press, 1974:47-9.

7 Channon S, Baker JE, Robertson MM. Working memory in clinical depression: an experimental study. Psychol Med 1993;23:87-91.

8 Channon S, Baker JE. Reasoning strategies in depression: effects of depressed mood on a syllogism task. Personal and effects of depressed mood on a syllogi
Individual Differences 1994:17:707-11.

9 Channon S. Executive dysfunction in depression: the Wisconsin Card Sorting Test. F Affective Disord 1996;39: 107-14.

10 Hartlage S, Alloy LB, Vazquez C, et al. Automatic and effortful processing in depression. Psychol Bull 1993;113: 247-78.

11 Teasdale JD, Barnard PJ. Affect, cognition and change. Hove: Lawrence Erlbaum Associates, 1993.

12 Watts FN. Problems of memory and concentration. In: CG Costello, ed. Symptoms of depression. New York: Academic Press, 1993:113-40.

13 Austin MP, Ross M, O'Carrol RE, et al. Cognitive function in major depression. 7 Affect Disord 1992;25:21-30.

14 Trichard C, Martinot M, Alagille MC, et al. Time course of frontal lobe dysfunction in severely depressed in-patients: a frontal lobe dysfunction in severely depressed in-patients: a 25:79-85.

15 Robertson G, Taylor PJ. Some cognitive correlates of affective disorders. Psychol Med 1985;15:297-309.

16 Calev A, Doron N, Chazan S. Retrieval from semantic memory using meaningful and meaningless constructs by depressed, stable bipolar and manic patients. Br f Clin Psychol 1989;28:67-73.

17 Watts FN, MacLeod A, Morris L. Associations between phenomenal and objective aspects of concentration problems in depressed patients. Br f Psychol 1988;79:241-50.

18 Elliott R, Sahakian BJ, McKay AP, et al. Neuropsychological impairments in unipolar depression: the influence of perceived failure on subsequent performance. Psychol Med 1996;26:975-89.

19 Channon S, Baker JE. Depression and problem-solving performance on a fault-diagnosis task. Applied Cognitive formance on a fault-diag
Psychology 1996;10:327-36.

20 Dobson DJD, Dobson KS. Problem-solving strategies in depressed and non-depressed college students. Cognitive depressed and non-depressed colle

21 Silberman EK, Weingartner H, Post RM. Thinking disorder in depression: logic and strategy in an abstract reasoning task. Arch Gen Psychiatry 1983;40:775-80.

22 Baker JE, Channon S. Reasoning in depression: impairment on a concept discrimination learning task. Cognition and Emotion 1995;9:579-97.
23 Channon S, Baker JE, Robertson MM. Effects of structure and clustering on recall and recognition memory in clinical depression. F Abnorm Psychol 1993;102:323-6.

24 Beats BC, Sahakian BJ, Levy R. Cognitive performance in tests sensitive to frontal lobe dysfunction in the elderly depressed. Psychol Med 1996;26:591-603.

25 Ellis HC, Ashbrook PW. Resource allocation model of the effects of depressed mood states on memory. In: K Fiedler, Forgas, eds. Affect, cognition and social behaviour. Gottingen: Hogrefe 1988: 25-43.

26 Hasher L, Zacks RT. Automatic and effortful processes in memory. F Exp Psychol Gen 1979;108:356-88.

27 Hertel PT. Depressive deficits in word identification and recall. Cognition and Emotion 1994;8:313-27.

28 Hertel PT, Hardin TS. Remembering with and without awareness in a depressed mood: evidence of deficits in iniawareness in a depressed mood: evidence
tiative. F Exp Psychol Gen 1990;119:5-59.

29 Richards PM, Ruff RM. Motivational effects on neuropsychological functioning: comparison of depressed versus nondepressed individuals. f Consult Clin Psychol 1989;57: 396-402.

30 Hertel PT, Rude SS. Depressive deficits in memory: focusing attention improves subsequent recall. F Exp Psychol Gen 1991;120:301-9.

31 Hertel PT, Milan S. Depressive deficits in recognition: dissociation of recollection and familiarity. $\mathcal{f}$ Abnorm Psychol 1994;103:736-42.

32 Hertel PT, Knoedler AJ. Solving problems by analogy: the benefits and detriments of hints and depressed moods. Memory and Cognition 1996;24:16-25.

33 Channon S, Daum I, Polkey CE. The effect of categorization on verbal memory after temporal lobectomy. Neuropsychologia 1989;27:777-85.

34 Burgess PW, Shallice T. Response suppression, initiation and strategy use following frontal lobe lesions. Neuropsychologia 1996;34:263-73.

35 Shallice T, Burgess PW. Deficits in strategy application following frontal lobe damage in man. Brain 1991;114:72741.

36 Kucera H, Francis WN. Computational analysis of present day American English. Providence, RI: Brown University Press, 1967.

37 American Psychiatric Association. Diagnostic and statistical manual of mental disorders, 3rd ed, revised. Washington, DC: APA, 1987.

38 Spitzer RL, Williams JBW, Gibbon M, et al. Structured clinical interview for DSM-III-R. Washington, DC: American Psychiatric Press, 1990.

39 Beck AT, Ward CH, Mendelson $\mathrm{M}$, et al. An inventory for measuring depression. Arch Gen Psychiatry 1961;4:561-71.

40 Hamilton M. Development of a rating scale for primary depressive illness. British fournal of Social and Clinical Psychology 1967;6:278-95.

41 Baddeley A, Emslie H, Nimmo Smith I. The speed and capacity of language-processing test. Bury St Edmunds, England: Thames Valley Test Company, 1992.

42 della Rocchetta AI, Milner B. Strategic search and retrieval inhibition: the role of the frontal lobes. Neuropsychologia 1993;31:503-24.

43 Gershberg FB, Shimamura AP. Impaired use of organizational strategies in free recall following frontal lobe damage. Neuropsychologia 1995;13:1305-33.

44 Shallice T, Fletcher P, Frith CD, et al. Brain regions associated with acquisition and retrieval of verbal episodic memory. Nature 1994;368:633-5.

45 Calev A, Irwin PG. Recall and recognition in depressives: uses of matched tasks. Br f Clin Psychol 1985;24:127-8.

46 Shallice T, Burgess PW. The domain of supervisory processes and temporal organization of behaviour. Phillo Trans R Soc Lond B Biol Sci 1996;351:1387-527. 